\title{
ENVELHECIMENTO, QUALIDADE DE VIDA E BEM-ESTAR SUBJETIVO: PERCEPÇÕES DE IDOSOS PARTICIPANTES DE UM GRUPO SOCIAL
}

\author{
Cristina de Fátima Ventura
}

Universidade Metodista de São Paulo, Brasil. cristinal@sp.senac.br

\begin{abstract}
Resumo. O objetivo desse estudo é retratar a experiência do envelhecimento a partir da perspectiva do próprio idoso, abordando a temática do Bem-Estar Subjetivo (BES) e Qualidade de Vida (QV) a luz da teoria lifespan. Estudo transversal de natureza qualiquantitativa, com 80 idosos de ambos os sexos, participantes de um grupo social da terceira idade de importante instituição brasileira, no município de Bertioga. Para coleta de dados, aplicou-se um questionário sociodemográfico e três instrumentos de natureza quantitativa, validados internacionalmente e uma entrevista semiestruturada, com análise pelo método hermenêutico-dialético. Apesar de se tratar de resultados preliminares, é possível afirmar que há uma preocupação dos idosos com a manutenção de vida ativa e independente, demonstrando que estar engajado em atividades sociais e de lazer, sentindo-se pertencente a um grupo e respeitado pela sociedade é de fundamental importância para sua avaliação de satisfação com a vida. Espera-se que o estudo possa contribuir com um novo olhar para o desenvolvimento humano, considerando a subjetividade presente no envelhecimento, uma vez que as questões relativas a esse processo são complexas e exigem uma maior atenção para criação e cumprimento de políticas com foco na promoção da saúde.
\end{abstract}

Palavras-Chave: Envelhecimento; Qualidade de Vida; Idoso; Bem-Estar Subjetivo.

AGING, QUALITY OF LIFE AND SUBJECTIVE WELL-BEING: PERCEPTIONS OF ELDERLY PARTICIPANTS IN A SOCIAL GROUP.

Abstract. The aim of this study is to portray the experience of aging from the perspective of the elderly, addressing the theme of Subjective Well-Being (SWB) and Quality of Life (QOL) in the light of the lifespan theory. The Cross-sectional study of a qualitative and quantitative nature was conducted with 80 elderly people of both sexes, participating in a social group of the elderly within an important Brazilian institution, in the city of Bertioga. For data collection, a sociodemographic questionnaire and three instruments of a quantitative nature were applied, internationally validated and a semi-structured interview, with analysis by the hermeneutic-dialectic method. Despite being preliminary results, it is possible to affirm that elderly people are concerned with the maintenance of an active and independent life, demonstrating that being engaged in social and leisure activities, feeling belonging to a group and respected by society is of fundamental importance for their life satisfaction assessment. It is hoped that the study can contribute with a new look at human development, considering the subjectivity present in aging, since the issues related to this process are complex and require greater attention to the creation and enforcement of policies focused on promotion of health.

Keywords: Aging; Quality of Life; Elderly; Subjective Well-Being.

\section{INTRODUÇÃO}

No século XXI, com o aumento da expectativa média de vida no mundo, a velhice transformou-se em tema social de grande relevância trazendo à tona a necessidade de novos olhares para o processo de envelhecimento, com necessidade de novas pesquisas e 
maior atenção para criação de políticas que promovam a saúde e que contribuam para a manutenção da autonomia e valorizem as redes de suporte social para uma vida mais digna.

No cenário global, os países desenvolvidos, com maior destaque os europeus, foram os primeiros a experimentar a questão da longevidade, pois tinham e tem condições mais favoráveis sob o ponto de vista dos aspectos econômicos, sociais e biológicos, possibilitando o envelhecimento de forma lenta e gradual.

Segundo dados da Organização das Nações Unidas (ONU,2002), no período de 1970 a 2000, foi observado um crescimento do envelhecimento populacional de 54\% nos países desenvolvidos, enquanto que para os países em desenvolvimento, no mesmo período, foi observado um crescimento de $123 \%$. No Brasil, um país em desenvolvimento, esse crescimento acelerado é confirmado pelo Instituto Brasileiro de Geografia e Estatística (IBGE, 2013), onde registra-se que a esperança de vida ao nascer do brasileiro, aumentou de 45,5 anos em 1940 para 76,3 anos em 2018, colocando o Brasil na 69ำ posição no ranking mundial de esperança de vida, fato explicado por esse mesmo Instituto pelo avanço da medicina, declínio da taxa de mortalidade e da taxa de fecundidade, aumento do saneamento básico, e também por mais acesso a informação por parte da população.

A Organização Mundial de Saúde (OMS,2005) define o idoso como aquele indivíduo com 60 anos de idade ou mais, limite válido para os países em desenvolvimento e 65 anos de idade para os países desenvolvidos. A OMS, com o intuito de criar referências de amparo aos idosos com vistas a substituir os modelos curativos, baseados nas doenças, para os modelos centrados na saúde, estabelece o conceito de Envelhecimento Saudável, definindo-o como "o processo de desenvolvimento e manutenção da capacidade funcional que permite o bem-estar em idade avançada" (p.13). Posteriormente já objetivando transmitir uma mensagem mais abrangente reconhecendo que além da saúde, outros fatores interferem na forma como as pessoas envelhecem, adota o termo envelhecimento ativo, entendido como "a otimização das oportunidades de saúde, participação e segurança, com o objetivo de melhorar a qualidade de vida à medida que as pessoas ficam mais velhas" (p.15).

Atualmente, os especialistas no estudo do envelhecimento referem-se a três grupos de pessoas mais velhas: os idosos jovens, os idosos velhos e os idosos mais velhos. O termo idoso jovens geralmente se refere a pessoas de 60 a 74 anos, que costumam estar ativas, 
cheias de vida e vigorosas. Os idosos velhos, de 75 a 84 anos, e os idosos mais velhos, de 85 anos ou mais, são aqueles que têm maior tendência para a fraqueza e para a enfermidade, e podem ter dificuldade para desempenhar algumas atividades da vida diária (Papalia \& Feldman,2013).

De acordo com teoria lifespan (Baltes,1987), corrente dominante na Psicologia do Envelhecimento, o desenvolvimento do ser humano ocorre ao longo da vida e implica em dois aspectos importantes. O primeiro consiste na ideia geral de que o desenvolvimento se estende por toda vida útil da pessoa e o segundo, considera a questão de que o desenvolvimento ao longo da vida envolve processos de mudança que não se originam no nascimento, mas que também ocorrem em períodos posteriores a esse. Consiste na ocorrência de ganhos e perdas, numa relação dinâmica e não linear, contribuindo para a quebra do paradigma de que a velhice estaria sempre associada a perdas. Tal conceito, propõe uma revolução no estudo do envelhecimento humano pois parte da premissa que todas as fases do ciclo de vida contribuem igualmente na regulação da natureza do desenvolvimento e nenhum período tem maior importância que outro. Consiste em um processo multidirecional que supera a perspectiva de crescimento e declínio e com múltiplas dimensões influenciadas por aspectos biológicos, sociais e psicológicos.

$\mathrm{Na}$ atualidade, são crescentes as discussões que trazem a preocupação com a Qualidade de Vida no processo de Envelhecimento. A crescente preocupação com o tema vem de um movimento dentro das ciências humanas e biológicas no sentido de valorizar parâmetros mais amplos que o controle de sintomas, a diminuição da mortalidade ou o aumento da expectativa de vida (Fleck, Louzada, Xavier, Chachamovick, Vieira, Santos \& Pinzon, 1999). A literatura científica ressalta que o construto Qualidade de Vida é complexo e que admite vários significados, com variadas abordagens teóricas (Kimura \& Silva, 2010). Contudo, há uma concordância entre os autores com relação a subjetividade, valorizando as singularidades considerando as percepções dos indivíduos e a multidimensionalidade como inerentes ao construto. Dessa forma, nota-se o quanto QV é um tema difícil de se avaliar apenas com métodos quantitativos, pois cada pessoa tem uma percepção própria. A OMS (2005) define QV como "a percepção do indivíduo acerca de sua posição na vida, no contexto de sua cultura e no sistema de valores em que vive e em relação a suas expectativas, seus padrões e suas preocupações" (p.14). A definição reflete a natureza subjetiva da avaliação que está imersa no contexto cultural, social e de meio ambiente. $O$ que está em questão não é a natureza objetiva do meio ambiente, do estado funcional ou do 
estado psicológico, ou ainda como o profissional de saúde ou um familiar avalia essas dimensões: é a percepção do próprio indivíduo. Trata-se de uma definição que resulta de um consenso internacional, representando uma perspectiva transcultural, bem como multidimensional, que contempla a complexa influência da saúde física e psicológica, nível de independência, relações sociais, crenças pessoais e das suas relações com características salientes do respectivo meio na avaliação subjetiva da qualidade de vida individual.

Outro tema que está presente no processo de envelhecimento saudável é o Bem-Estar Subjetivo (BES), conceito científico desenvolvido por Diener (1984), que destaca três aspectos importantes: o primeiro é a subjetividade onde o bem-estar reside dentro da experiência do indivíduo; o segundo consiste no entendimento de que bem-estar não é apenas a ausência de fatores negativos, mas também a presença de fatores positivos; e o terceiro salienta que o bem-estar inclui uma medida global ao invés de somente uma medida limitada de um aspecto da vida. Possui pelo menos dois componentes: cognição e afeto. $O$ construto BES, diz respeito a como e por que as pessoas experienciam suas vidas positivamente e como avaliam subjetivamente a sua qualidade de vida.

O estudo se propõe a mensurar a Qualidade de Vida (QV) e o Bem-Estar Subjetivo (BES) dos participantes deste grupo social, assim como investigar a experiência do envelhecimento, através da autopercepção dos idosos, buscando compreender até que ponto o prolongamento da vida vem acompanhado de níveis de satisfação ou insatisfação e quais fatores são preditores para uma vida longeva e com qualidade.

\section{METODOLOGIA}

O presente estudo, de abordagem qualiquantitativo, descritivo de caráter transversal, foi realizado no município de Bertioga em São Paulo - Brasil.

A integração dos dados quantitativos e qualitativos já se configura como uma tendência crescente na área de saúde e a escolha da abordagem mista, teve como princípio a necessidade de explicitar o caráter subjetivo dos objetos de estudo, buscando compreender as perspectivas e significados dos sujeitos participantes, visando uma resposta mais ampliada do problema, mas que poderiam num primeiro momento receber as contribuições da perspectiva quantitativa para posterior aprofundamento. 
O projeto foi aprovado pelo Comitê de Ética em Pesquisa (parecer 2.960.584), em conformidade com o estabelecido na Resolução 466/12 e a fase de pesquisa de campo foi iniciada somente após a assinatura do Termo de Consentimento Livre e Esclarecido (TCLE) pelos sujeitos que concordaram em participar da mesma.

A amostra foi constituída por 80 sujeitos com 60 anos de idade ou mais, com média de idade de 67,9 anos, de ambos os sexos, sendo 59 mulheres e 21 homens, com escolaridade predominantemente do ensino superior, participantes de Grupo Social de Idosos de renomada instituição brasileira, reconhecida como pioneira em trabalhos com esse público, atuando desde 1963, promovendo o diálogo com as várias frentes da sociedade.

Os critérios de inclusão foram pessoas, matriculadas e ativas no Programa Trabalho Social com Idosos desta instituição, sem comprometimento cognitivo declarado com ausência de comprovação médica.

Na perspectiva quantitativa, aplicou-se um questionário sociodemográfico e foram utilizados três instrumentos reconhecidos internacionalmente, para coleta de dados que ocorreu entre os meses de maio a outubro de 2019, nas dependências da própria instituição, durante reunião mensal com os idosos com duração de 1 hora. Para mensurar qualidade de vida foram utilizados os instrumentos WHOQOL-Bref e WHOQOL-Old, com 4 temas divididos em 10 categorias, abordando o quanto o idoso tem tido certos sentimentos, o quanto se sente apto a fazer as coisas, o quanto se sente feliz e sobre relacionamento íntimo. A Escala de Bem-Estar Subjetivo, foi utilizada para mensurar satisfação com a vida (15 itens), afetos positivos (21) e afetos negativos (26), totalizando 62 ítens. Cada participante era identificado por um número, garantindo a confidencialidade das informações. Posterior a aplicação dos instrumentos foi realizada a análise dos dados com a utilização do software estatístico Statistical Package for Social Science (SPSS).

$\mathrm{Na}$ perspectiva qualitativa, posterior ao tratamento de dados da pesquisa quantitativa, os dados foram coletados por meio de entrevistas semiestruturadas, com questões abertas de acordo com a temática da pesquisa, por um único pesquisador, realizada com uma amostra de 8 idosos. Os idosos foram escolhidos de forma randômica, subdivididos em 2 categorias sendo, 4 idosos jovens com idade entre 60 e 74 anos e 4 idosos velhos com idade entre 75 e 84 anos.

O estudo qualitativo trabalha com o âmbito de significados, motivos, aspirações, crenças, 
valores e atitudes, o que equivale a uma zona mais profunda das relações, dos processos e dos fenômenos, por essa razão são pesquisas feitas geralmente com um número pequeno de entrevistados (Minayo, 2001).

As entrevistas foram realizadas em local reservado, retomando os objetivos do estudo, sendo preservada as identidades das participantes. Para isso, as entrevistas foram identificadas numericamente de acordo com a ordem da mesma.

O roteiro da entrevista considerou as seguintes perguntas:

1) O que é envelhecer bem para o Sr./ Sra.?

2) O quão (in)satisfeito você se considera com sua vida atualmente e o que lhe faz sentir dessa forma?

3) Se você pudesse mudar alguma coisa em sua vida, o que seria?

4) Como avalia sua qualidade de vida?

O registro da entrevista foi gravado em áudio e transcrito na íntegra pelo pesquisador e dado o direito ao entrevistado de escolher livremente o que seria mantido ou retirado do conteúdo.

Após o trabalho de campo, para a análise de dados, utilizou-se o modelo hermenêuticodialético, proposto por Minayo (2007), que leva o intérprete a entender o texto, a fala, o depoimento, como resultado de um processo social e de conhecimento com significado específico. A hermenêutica busca a compreensão e a dialética estabelece uma atitude crítica e, um ponto em comum entre as duas práticas, é que ambas consideram fundamentais as condições históricas do pensamento e partem do princípio de que o pesquisador é parte da realidade que investiga. Parte-se do princípio que não há investigador imparcial, uma vez que trata-se de pesquisa com seres humanos.

No princípio hermenêutico, que se constitui no estudo da interpretação de textos escritos, há a ordenação dos dados que inclui a transcrição das gravações, a classificação dos dados, momento em que estes foram embasados com a fundamentação teórica e constitui-se na leitura minuciosa após a transcrição de cada entrevista buscando os temas centrais e agregando-os em categorias. No princípio dialético para análise de dados qualitativos, a 
fala dos atores sociais é contextualizada para ser melhor compreendida, considerando que uma mesma linguagem pode ter diferentes significados.

\section{RESULTADOS}

Primeiramente foi realizado um descritivo estatístico dos dados demográficos caracterizando toda a amostra com posterior cruzamento considerando as variáveis idade, estado civil e situação financeira, subdivididas em 2 faixas etárias, seguindo a literatura internacional adotada neste estudo com idosos jovens entre 60 a 74 anos e idosos velhos entre 75 a 84 anos, com o objetivo de mensurar quantitativamente as questões de satisfação com a vida , afetos positivos e negativos relacionados ao Bem-Estar e a Qualidade de Vida.

A partir dessa informação estatística, iniciaram-se as entrevistas a fim de conhecer a subjetividade das experiências sob a ótica do idoso, explorando a multidimensionalidade do fenômeno envelhecimento e das várias direções que este se relaciona.

Por se tratar de resultados preliminares, ainda com um número limitado de dados, as primeiras análises permitem inferir que para o idoso, estar engajado em atividades sociais e de lazer e, se sentir pertencente à um grupo, é de fundamental importância para sua avaliação de satisfação com a vida. Nesse sentido o suporte de familiares é vital para esse sentimento também. Para Zimerman (2000), pertencer à um grupo permite a troca de afeto, carinho, de ideias, de sentimentos, de conhecimentos, de dúvidas.

A questão do respeito da sociedade com relação ao idoso e ao próprio processo de envelhecimento, sendo reconhecidos como seres atuantes e produtivos, fazem-se presentes no relato dos idosos como fator importante, carregado inclusive de alguma emoção. $\mathrm{Na}$ sociedade brasileira, o preconceito com relação às pessoas idosas ainda existe (as vezes de forma sutil ou até mesmo inconsciente), tornando-se um grande desafio a ser enfrentado. Para Rizzuto (2011), estereótipos em relação a idade contribuem para que pessoas mais velhas sintam que estão sendo marginalizadas pela sociedade, enquanto os mais jovens são favorecidos, pode ser prejudicial aos indivíduos mais velhos, influenciando sua confiança e percepção de suas habilidades para adquirir novos conhecimentos.

Independência financeira é outra questão destacada para o sentimento de envelhecer bem, demonstrando a preocupação com a pouca atenção do governo para as questões relativas a aposentadoria e serviços de saúde para essa população. Apesar deste cenário de 
incertezas, de forma geral há uma manifestação de que são felizes com o que tem, o que pode ser entendido em razão da maior parte do grupo já ser aposentado ou receber pensão.

Em contrapartida, a questão da autonomia relacionada a sua capacidade de tomar decisões e ter controle sobre sua própria vida aparece como fator preponderante para um processo de envelhecimento mais prazeroso e significativo, se comparado a questões de limitações físicas decorrente do declínio de mobilidade ou de alguma outra doença. Há uma preocupação dos idosos com a manutenção da vida ativa e independente, principalmente nos de idade mais avançada. Para Bowling \& Dieppe (2005), há ampla evidência de que muitos idosos se consideram felizes e bem, mesmo na presença de doença ou incapacidade.

A espiritualidade é outro componente com grande influência na população em questão, pois os idosos costumam ter uma ligação muito forte com religiosidade. A fé é um instrumento que os fortalece e capacita no sentido de apoio, conforto e esperança, sendo entendida como uma necessidade básica que as impulsiona na trajetória de suas vidas. Os resultados apontam que não há uma preocupação com a morte, mas sim com a incapacidade física e a possibilidade de ficar doente, sofrer e depender de alguém para cuidados básicos.

\section{CONSIDERAÇÕES FINAIS}

Envelhecer é uma experiência heterogênea, resultado de uma construção que o indivíduo faz durante toda a vida, portanto é um processo subjetivo e nunca será igual para todos.

A realização da entrevista semiestruturada, foi de fundamental importância para o estudo da percepção de QV e BES, pois a partir dos relatos foi possível compreender o significado da velhice para o sujeito.

Os achados apresentaram semelhanças entre aquilo que os entrevistados consideraram importante para a obtenção da vida com qualidade e questões abordadas por instrumentos que se destinam à quantificação de qualidade de vida e bem-estar, o que valida a abordagem adotada.

Espera-se que este estudo possa apresentar reflexões sobre a subjetividade do indivíduo idoso, contribuindo para o desenvolvimento e atualização das políticas públicas com a finalidade de garantir não só uma existência longeva, mas sobretudo mais saudável e feliz. Para além das políticas públicas, que no Brasil não são percebidas como efetivas, indicando 
um longo caminho a ser percorrido com relação a luta pelos direitos dessa parcela da população, torna-se imprescindivel o olhar humanizado para o processo de envelhecimento na sociedade contemporânea.

\section{REFERÊNCIAS}

Baltes, P. B. (1987). Theorical propositions of life-span developmental psychology: on hte dynamics growth and decline. Developmental Psychology, 32 (5), 611-626.

Bowling, A., \& Dieppe, P. (2005). What is successful aging: perspectives from the behavioral sciences. New York: Cambridge University.

Diener, E. (1984). Subjective well-being. Psychological Bulletin, v. 95, 542-575.Fleck, M.P.A., Louzada, S., Xavier, M., Chachamovick, E., Vieira, G., Santos, L., \& Pinzon, V. (1999).

Desenvolvimento da versão em português do instrumento de avaliação de qualidade de vida da OMS (WHOQOL-100) Development of the Portuguese version of the OMS evaluation instrument of quality of life. Revista Brasileira de Psiquiatria, 21.

Instituto Brasileiro de Geografia e Estatística (2013). Atlas do Censo Demográfico 2010. Rio de Janeiro: Instituto Brasileiro de Geografia e Estatística - IBGE.

Kimura, M., \& Silva, J. V. (2010). Índice de Qualidade de Vida de Ferrans e Powers. Rev. Esc Enferm.USP, 43 (Esp), 1098-1104. Recuperado em 01 julho, 2015. www.scielo.br/ v43nspe/a14v43ns.pdf.

Minayo, M.C.S. (2001). Pesquisa Social. Teoria, método e criatividade, 18.ed., Petrópolis: Vozes.

Minayo, M.C.S. (2007). O desafio do conhecimento: pesquisa qualitativa em saúde,10. ed., São Paulo (SP): Hubitec/Abrasco.

Organização das Nações Unidas (2002). Plano de Ação Internacional para Envelhecimento.

Papalia, D. E., \& Feldman, R. D. (2013). Desenvolvimento Humano, 12a․ed. Porto Alegre: Artmed.

Rizzuto, T. E. (2011). Age and technology innovation in the workplace: does work context matter? Computer in Human Behavior, v.27, n.5, 1612-1620.

The WHOQOL Group (1995). The World Health Organization Quality of life Assessment: position paper from the World Health Organization. Soc. Sci. Med, 41, n.10.

World Health Organization (2005). Envelhecimento ativo: uma política de saúde. Brasília:Organização Panamericana de Saúde.

Zimerman, G. I. (2000). Velhice: aspectos biopsicossociais. Porto Alegre: Artmed. 